Published in final edited form as:

Nat Genet. 2010 September ; 42(9): 739-741. doi:10.1038/ng.639.

\title{
Genome-wide association analyses identifies a susceptibility locus for tuberculosis on chromosome 18q11.2
}

\author{
Thorsten Thye ${ }^{\# 1,2}$, Fredrik O. Vannberg ${ }^{\# 3}$, Sunny H. Wong ${ }^{3}$, Ellis Owusu-Dabo ${ }^{4,5}$, Ivy Osei ${ }^{6}$, \\ John Gyapong ${ }^{6}$, Giorgio Sirugo ${ }^{7}$, Fatou Sisay-Joof ${ }^{7}$, Anthony Enimil ${ }^{4,8}$, Margaret A. \\ Chinbuah $^{6}$, Sian Floyd ${ }^{9}$, David K. Warndorff ${ }^{10}$, Lifted Sichali ${ }^{10}$, Simon Malema ${ }^{10}$, Amelia C. \\ Crampin $^{9}$, Bagrey Ngwira ${ }^{10}$, Yik Y. Teo ${ }^{3}$, Kerrin Small ${ }^{3}$, Kirk Rockett ${ }^{3}$, Dominic \\ Kwiatkowski $^{3}$, Paul E. Fine ${ }^{9,10}$, Philip C. Hill ${ }^{7}$, Melanie Newport ${ }^{7}$, Christian Lienhardt ${ }^{7}$, \\ Richard A. Adegbola ${ }^{7}$, Tumani Corrah ${ }^{7}$, Andreas Ziegler ${ }^{2}$, African TB Genetics \\ Consortium ${ }^{12}$, Wellcome Trust Case Control Consortium ${ }^{12}$, Andrew P. Morris ${ }^{3}$, Christian G.

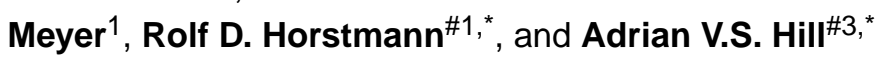 \\ ${ }^{1}$ Department of Molecular Medicine, Bernhard Nocht Institute for Tropical Medicine, Hamburg, \\ Germany \\ ${ }^{2}$ Institute of Medical Biometry and Statistics, University Hospital Schleswig-Holstein, Campus \\ Lübeck, Lübeck, Germany \\ ${ }^{3}$ Wellcome Trust Centre for Human Genetics, University of Oxford, United Kingdom \\ ${ }^{4}$ Kumasi Centre for Collaborative Research in Tropical Medicine, Kumasi, Ghana \\ ${ }^{5}$ Department of Community Health, College of Health Sciences, Kwame Nkrumah University of \\ Science and Technology, Kumasi, Ghana \\ ${ }^{6}$ Health Research Unit, Ghana Health Service, Accra, Ghana \\ ${ }^{7}$ MRC Laboratories, Fajara, Banjul, The Gambia \\ ${ }^{8}$ Komfo Anokye Teaching Hospital, Kumasi, Ghana \\ ${ }^{9}$ London School of Hygiene and Tropical Medicine, London, United Kingdom \\ ${ }^{10}$ Karonga Prevention Study, Chilumba, Karonga District, Malawi \\ \# These authors contributed equally to this work.
}

\footnotetext{
Users may view, print, copy, download and text and data- mine the content in such documents, for the purposes of academic research, subject always to the full Conditions of use: http://www.nature.com/authors/editorial_policies/license.html\#terms

*Correspondence should be addressed to Rolf D. Horstman (horstmann@bnitm.de) and Adrian V.S. Hill (adrian.hill@well.ox.ac.uk). ${ }^{12}$ For Consortia membership see Supplementary Note.

Author Contributions: T.T., F.O.V., S.H.W., C.G.M., R.D.H., A.S.V.H. wrote the paper. T.T., F.O.V., S.H.W. performed the genotyping. T.T., F.O.V., S.H.W. performed the analysis. T.T., F.O.V., S.H.M., Y.Y.T., K.S. contributed to the statistical methods. E.O.D and J.G. led sample procurement and supervision of field work in Kumasi and Accra, respectively. T.T., F.O.V., S.H.W., I.O., P.C.H., G.S.J., A.E., M.A.C., S.F., D.K.W., L.S., S.M., A.C.C., B.N., K.R., D.K., P.E.F., G.S., M.N., C.L., R.A.A., T.C., A.Z., A.P.M., C.G.M., R.D.H., A.S.V.H. contributed to the experimental design and collection of the sample cohorts. All authors critically reviewed the manuscript. T.T., F.O.V., C.G.M., R.D.H. and A.V.S.H. led the research effort.

URLs: The International HapMap Project (http://hapmap.ncbi.nlm.nih.gov); The Wellcome Trust Case Control Consortium (www.wtccc.uk.org)

Competing interests statement: The authors declare that they have no competing financial interests.
} 


\section{Abstract}

We combined two tuberculosis (TB) genome-wide association studies (GWAS) from Ghana and The Gambia with subsequent replication totalling 11,425 participants. A significant association with disease was observed at SNP rs4331426 located in a gene-poor region on chromosome $18 \mathrm{q} 11.2\left(P=6.8 \times 10^{-9}, \mathrm{OR}=1.19,95 \% \mathrm{CI}=1.13-1.27\right)$. Our finding shows that GWAS can identify novel loci for infectious causes of mortality even in Africa where levels of linkage disequilibrium are particularly low.

Tuberculosis (TB) causes significant morbidity and mortality world-wide ${ }^{1,2}$ with a high disease burden in sub-Saharan Africa. Although previous studies have indicated that susceptibility to pulmonary TB has a substantial genetic component ${ }^{3,4}$, progress in the determination of contributing genetic variants has been slow. While genome-wide association studies (GWAS) have successfully identified many common variants associated with a variety of diseases ${ }^{5}$, studies of infectious diseases have so far largely failed to identify novel causative variants, partly due to the small sample sizes studied ${ }^{6,7}$. We here present a combined analysis of two West African genome-wide association studies, a new data set from Ghana and The Wellcome Trust Case Consortium (WTCCC) tuberculosis study of Gambians (WTCCC, unpublished) with further replication series analyzing in all 11,425 African individuals.

The two GWA studies were performed with TB cases and controls recruited from Ghana and The Gambia (study flow-chart in Supplementary Methods) The Ghanaian GWA study included 921 cases and 1,740 controls genotyped using the Affymetrix SNP Array 6.0 with 743,635 autosomal SNPs included in the analysis (Supplementary Methods). The Gambian WTCCC GWA study included 1,316 cases and 1,382 controls who were genotyped using the Affymetrix GeneChip 500K array with 354,607 autosomal SNPs (Supplementary Methods) (WTCCC, unpublished). In total, 333,754 SNPs were included in the combined analysis of 2,237 cases and 3,122 controls from Ghana and The Gambia, achieving 90\% power to detect significant association at genotype relative risks of 1.4 or greater. Multidimensional scaling (MDS) analysis was carried out to investigate the population structure (Supplementary Methods $)^{8}$. The first six MDS components were incorporated as covariates into the logistic regression association analysis in the combined study. The subsequent quantile-quantile plot showed inflation at a level comparable to other GWA studies after correction with the MDS components ( $\lambda=1.05$, Supplementary Methods).

In order to look at these findings more globally we subjected the Ghanaian and Gambian results to an MDS analysis, including available data from the Nigerian Yoruba population, and also non-African populations (Han Chinese, Japanese, CEPH) from the HapMap Project. As expected, differences to the Chinese/Japanese and CEPH populations were large, but the three populations originating from West Africa could also be distinguished clearly (Supplementary Methods). These population-specific differences complicate accurate imputation of the Ghanaian or Gambian genotypes using the Nigerian Yoruba HapMap data set. Fst values for genetic distances for each pair of Ghanaian populations, Gambian populations and between Ghanaian and Gambian populations as a whole are given in Supplementary Methods. 
In the combined analysis, we identified 17 SNPs with $P \unlhd 0^{-5}$ with the same direction of effect in both the Ghanaian and WTCCC Gambian study groups (Supplementary Table 1). We attempted to replicate these 17 SNPs in an additional 1,076 cases and 1,611 controls from Ghana (Replication I). The addition of the results obtained in Replication I to the two GWA studies revealed two SNPs (rs2335704 and rs4331426) with $P<5 \times 10^{-7}$

(Supplementary Table 1). To corroborate the findings, we further genotyped these two SNPs in additional cohorts from Ghana (150 cases, 2,214 controls) and Malawi (236 cases, 779 controls), (Replication II), as well as in 332 family trios/duos from Ghana, whereby cases of nuclear families were part of the complete association analysis. For the two hit regions, neighbouring SNPs were studied in an attempt to fine-map the association signals (Supplementary Table 2).

When combining the GWA study data with those from Replications I and II, SNP rs4331426, which maps to chromosome 18q11.2, obtained the highest association signal with an overall $P=6.8 \times 10^{-9}(\mathrm{OR}=1.19,95 \% \mathrm{CI}=1.13-1.27)$, or $P_{\mathrm{GC}}=1.6 \times 10^{-8}$ after genomic control correction (Fig. 1, Table 1). Consistent estimates of ORs as assessed by the Breslow Day test were observed $(P=0.95)$ across the studies and ethnic groups, and no difference in ORs was seen between the logistic regression and Mantel-Haenszel tests. Heterogeneity between the studies was negligible with $I^{2}=0.0 \%$. The analysis of Ghanaian nuclear families supported the association, $P=0.016(\mathrm{OR}=1.33,95 \% \mathrm{CI}=1.05-1.68)$, although this statistic was not included in the final $P$ value because the cases were part of the association analyses (Table 1).

The associated chromosome 18 variant (SNP rs4331426) is common in African populations but much rarer in all other populations and has a remarkably consistent OR across African cohorts, including the East African samples from Malawi. Additional work is required to ascertain the causative variant, its functional significance and any possible counterbalancing selective pressure. The nearest genes to this SNP are GATA6, CTAGE1, RBBP8, CABLES1, as well as a number of, as yet, un-annotated open reading frames. However, the generally low linkage disequilibrium on 18q11.1-q11.2 suggests that rs4331426 is within a genedesert region that is punctuated by evolutionarily conserved domains with regulatory potential.

In addition to rs4331426, a second variant, rs2335704 on chromosome 2 was found associated after Replication $\mathrm{I}\left(P=3 \times 10^{-7}, \mathrm{OR}=1.23,95 \% \mathrm{CI}=1.14-1.34\right)$. However, the significance decreased to $P=2.1 \times 10^{-6}(\mathrm{OR}=1.29,95 \% \mathrm{CI}=1.11-1.28)$ after adding results of Replication II (Supplementary Fig. 1, Supplementary Table 3). The Mantel-Haenszel test, stratified for ethnic groups, revealed heterogeneity in ORs between the Ghanaian ethnic group of the Ga-Adangbe compared to all other ethnicities. This heterogeneity suggests that the result should be interpreted with caution.

GWA studies in African populations are, in general, limited by extensive genetic diversity and shorter LD ranges, and to date no novel loci of genome wide statistical significance $\left(P<5 \times 10^{-8}\right)$ have been reported using the GWA approach in African studies (WTCCC, unpublished). A recent malaria GWA study in The Gambia found substantial population 
complexity ${ }^{6}$, indicating marked diversity among African ethnicities and emphasizing the need for careful population structure correction and ethnicity stratification.

In general, finding convincing non-MHC susceptibility loci across populations for infectious diseases has been difficult, even in individuals of European ancestry. A recent GWA study on HIV viral set-point revealed strong signals within the $H L A-B$ and $H L A-C$ loci, but not any novel non- $M H C$ loci $^{7}$. Pathogen variation may underlie some of the difficulties in finding loci for a given infectious disease and M. tuberculosis displays substantial geographic variation in genotype frequencies ${ }^{9}$. However, as we have shown previously and also here ${ }^{9-13}$, combined analyses with increased aggregate sample sizes may help in the identification of novel genetic variants, perhaps particularly those less sensitive to pathogen variation. Analyzing African individuals poses specific challenges, and genetic differences between populations, even within West Africa, are large enough to complicate standard imputation procedures. Assessing imputation accuracy using the YRI as reference we found a mean genotype error rate across all chromosomes of $8.2 \%$ for the combined Ghanaian and Gambian sample, which raises some concern about the validity of imputation in this setting. We performed a genome wide imputation analysis with stringent quality criteria (Rsq $>0.7$ ) followed by association analysis of the imputed SNPs (Supplementary Table 4). In these calculations, no variant reached a significance of $P<10^{-6}$. However, many variants did reach the significance level of the rs4331326 SNP, and these will be carried forward in future studies.

We analyzed variants of the Affymetrix SNP arrays located in candidate genes previously found to be associated with resistance or susceptibility to TB. We observed weak to moderate signals at several genes including the $H L A-D Q$ region (rs9469220, $P=0.0017$ ) (Supplementary Table 5). Considering the a priori associations for these regions there appears to be an over-representation of nominally significant results, suggesting that a number of the analyzed SNPs might be true TB susceptibility loci. However, more work will be necessary to replicate positive findings of previous association studies and to identify the true causative variants of these candidate gene analyses.

This work demonstrates that a novel non-MHC locus can be identified for a major fatal infectious disease caused by a highly polymorphic pathogen and suggests that many further loci may be identifiable with GWA studies of sufficient sample size, even in African populations, who suffer the greatest burden of communicable diseases.

\section{Supplementary Material}

Refer to Web version on PubMed Central for supplementary material.

\section{Acknowledgements}

We would like to thank the patients and families, field workers, nurses and physicians who contributed to these studies. We thank Dr Mark McCarthy and Dr Chiea Khor for critical assessment of the manuscript. 


\section{References}

1. The World Health Organization. Crunch time for tuberculosis control. Lancet. 2009; 373:1145. [PubMed: 19345812]

2. The World Health Organization. WHO annual report on global TB control--summary. Wkly Epidemiol. Reports. 2003; 78:122-8. [PubMed: 12747238]

3. Comstock GW. Tuberculosis in twins: a re-analysis of the Prophit survey. Am. Rev. Respir. Dis. 1978; 117:621-4. [PubMed: 565607]

4. Stead WW, Senner JW, Reddick WT, Lofgren JP. Racial differences in susceptibility to infection by Mycobacterium tuberculosis. N. Engl. J. Med. 1990; 322:422-7. [PubMed: 2300105]

5. WTCCC. Genome-wide association study of 14,000 cases of seven common diseases and 3,000 shared controls. Nature. 2007; 447:661-78. [PubMed: 17554300]

6. Jallow M, et al. Genome-wide and fine-resolution association analysis of malaria in West Africa. Nat. Genet. 2009

7. Fellay J, et al. A whole-genome association study of major determinants for host control of HIV-1. Science. 2007; 317:944-7. [PubMed: 17641165]

8. Ziegler A. Genome-wide association studies: quality control and population-based measures. Genet. Epidemiol. 2009; 33:S45-50. [PubMed: 19924716]

9. Gagneux S, et al. Variable host-pathogen compatibility in Mycobacterium tuberculosis. Proc. Natl. Acad. Sci. 2006; 103:2869-2873. [PubMed: 16477032]

10. Fitness J, et al. Large-scale candidate gene study of tuberculosis susceptibility in the Karonga district of northern Malawi. Am. J. Tro. Med. Hyg. 2004; 71:341-9. [PubMed: 15381817]

11. Lienhardt $\mathrm{C}$, et al. Investigation of environmental and host-related risk factors for tuberculosis in Africa. I. Methodological aspects of a combined design. Am. J. Epidemiol.. 2002; 155:1066-73. [PubMed: 12034586]

12. Thye $\mathrm{T}$, et al. MCP-1 promoter variant $-362 \mathrm{C}$ associated with protection from pulmonary tuberculosis in Ghana, West Africa. Hum. Mol. Genet. 2009; 18:381-8. [PubMed: 18940815]

13. Intemann CD, et al. Autophagy gene variant IRGM -261T contributes to protection from tuberculosis caused by Mycobacterium tuberculosis but not by M. africanum strains. PLoS Pathog. 2009; 5:e1000577. [PubMed: 19750224] 


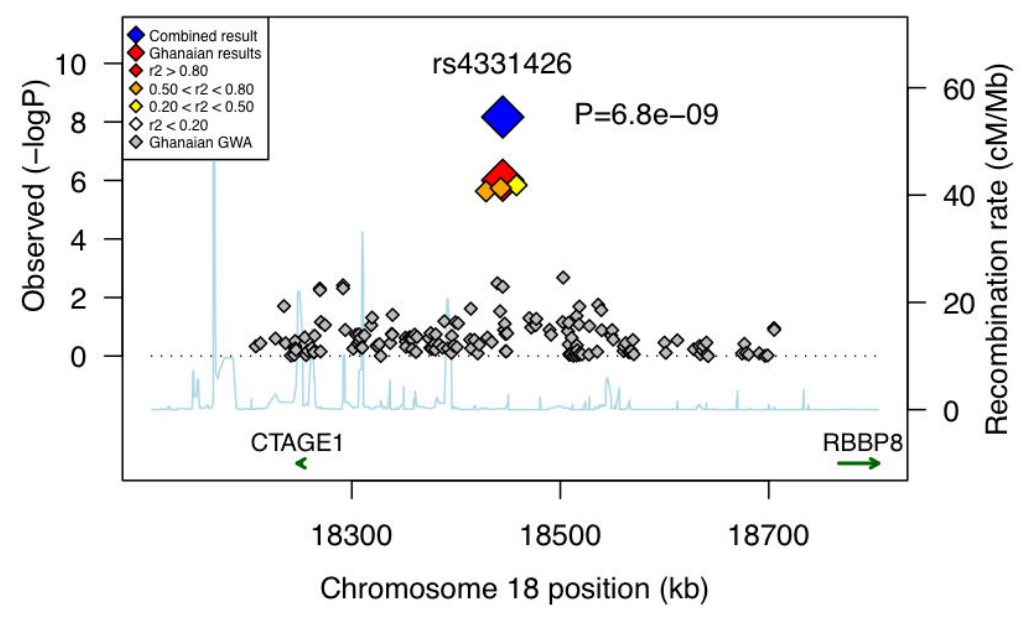

Figure 1.

Association plot with fine mapping markers on chromosome 18 in the combined analysis $\left(r^{2}\right.$ values between rs4331426 and adjacent SNPs derived from the Ghanaian population; $P$ uncorrected for $\lambda_{\mathrm{GC}}$ ). 
Table 1

Association statistics of rs 4331426 in the combined analysis

\begin{tabular}{|c|c|c|c|c|c|c|}
\hline rs4331426 - G allele & Controls & Controls & Cases & Cases & OR 95\% CI & $P$ value \\
\hline GWA study scan & $\boldsymbol{N}$ & Freq & $\boldsymbol{N}$ & Freq & & \\
\hline Ghana & 1740 & 0.448 & 921 & 0.491 & $1.18(1.05-1.32)$ & $4.3 \mathrm{E}-03$ \\
\hline The Gambia (WTCCC) & 1377 & 0.476 & 1309 & 0.521 & $1.18(1.06-1.31)$ & $2.9 \mathrm{E}-03$ \\
\hline Replication I & & & & & & \\
\hline Ghana & 1609 & 0.429 & 1076 & 0.477 & $1.19(1.06-1.33)$ & $2.8 \mathrm{E}-03$ \\
\hline Replication II & & & & & & \\
\hline Ghana & 2199 & 0.442 & 148 & 0.476 & $1.18(0.92-1.51)$ & $1.9 \mathrm{E}-01$ \\
\hline Malawi & 576 & 0.525 & 178 & 0.563 & $1.15(0.91-1.45)$ & $2.3 \mathrm{E}-01$ \\
\hline Combined analysis & 7501 & & 3632 & & $1.19(1.12-1.26)$ & $6.8 \mathrm{E}-09$ \\
\hline$\lambda_{\text {GC }}$ corrected combined $P$ value & & & & & & $1.6 \mathrm{E}-08$ \\
\hline Ghanaian nuclear families ${ }^{*}$ & & & & & $1.33(1.05-1.68)$ & $1.6 \mathrm{E}-02$ \\
\hline
\end{tabular}

* Cases from nuclear families were part of the Ghanaian GWA case-control study; the TDT statistic is not included in the combined analysis. 